\section{Levantamento de dados sobre o conhecimento e informação acerca dos medicamentos genéricos em uma população de pacientes do serviço de saúde ambulatorial do Recife, Pernambuco, Brasil}

\author{
Knowledge and information concerning generic \\ drugs in a public outpatient population in Recife, \\ Pernambuco, Brazil
}

\footnotetext{
${ }^{1}$ Centro de Ciências Biológicas e da Saúde, Universidade Tiradentes, Aracaju, Brasil.

2 Centro de Ciências da Saúde, Universidade Federal de Pernambuco, Recife, Brasil.

Correspondência C. E. Rocha

Centro de Ciências Biológicas e da Saúde, Universidade Tiradentes.

Rua Deputado Clóvis Rollemberg 300A, Aracaju, SE 49037-120, Brasil.

chiaraerocha@terra.com.br
}

\begin{abstract}
Brazilian Federal Act 9,787/99, regulating generic medicines, includes rules for drug registration and provides for the population's access to safe, effective, high-quality, and low-cost medication. Four years after the introduction of generic drugs on the Brazilian market, we proposed to measure knowledge and information on generic drugs among the clientele at a public outpatient clinic in Recife, Pernambuco State, applying a questionnaire in 2000. 76.1\% of the interviewees were women, 95.7\% had heard of generic drugs, and $68.1 \%$ could define generic medication. Government action to publicize the properties of such medicines and their advantages for consumers has thus reached the vast majority of the population.
\end{abstract}

Generic Drugs; Knowledge; Information; Ambulatory Health Services
Chiara Erminia da Rocha ${ }^{1}$

José Augusto Cabral de Barros 2

Maria Dolores Paes Silva 2

\section{Introdução}

O medicamento genérico é aquele que pode ser produzido livremente, vencido o prazo de proteção patentária do produto de marca comercial, devendo ser semelhante ao de marca em bioequivalência e biodisponibilidade a fim de obter o mesmo efeito terapêutico ${ }^{1}$.

No Brasil, os gastos com saúde representam o quarto maior grupo de despesas familiares, sendo o primeiro habitação. Dentre estes gastos, 66 $70 \%$ das despesas familiares se devem aos medicamentos e planos de saúde. Salientemos que a população carente gasta mais com medicamentos quando comparada à parcela mais rica que direciona recursos também para o pagamento dos planos de saúde 2 .

É de conhecimento geral que os países mais pobres não fazem parte do mercado de medicamentos na forma como este está estabelecido nos países desenvolvidos, sendo necessário a existência do compromisso público e de uma intervenção governamental a fim de que se assegure o acesso igualitário e universal às ações de assistência à saúde entre as quais se inclui a assistência farmacêutica ${ }^{3,4}$.

Em 1998, a Portaria no. 3.9165 aprova a Política Nacional de Medicamentos que tem por objetivo garantir a segurança, qualidade e eficácia dos medicamentos, propiciar o uso racional dos mesmos e o acesso universal e igualitário aos medicamentos essenciais. Para o alcance dessas 
metas, sucintamente, tem-se o propósito de estabelecer a relação de medicamentos essenciais, reorientação da assistência farmacêutica, estímulo à produção de medicamentos pelos laboratórios oficiais e a sua regulamentação sanitária.

A Política Nacional de Medicamentos propõe mudanças que poderão atender aos interesses dos usuários desses produtos. Uma dessas propostas é o estímulo à produção de medicamentos genéricos pelas indústrias nacionais a fim de diminuir os gastos com a aquisição de fármacos, promover a concorrência entre os produtos já existentes no mercado e promover o uso racional dos medicamentos a partir de ações junto ao médico, ao farmacêutico e à sociedade 5 .

Inquérito, realizado em 2001 pela Agência nacional de Vigilância Sanitária (ANVISA) 6 , revelou que do total de 2.200 entrevistados, $46 \%$ adquirem medicamentos via receita médica, porém, destes $\mathbf{8 0} \%$ continham medicamentos prescritos segundo o seu nome de marca comercial em detrimento dos $9 \%$ que continham somente o medicamento genérico. Nesta mesma pesquisa, $51 \%$ do total de consumidores nunca obtiveram informação a respeito do medicamento genérico pelo seu médico.

A Lei no. 9.787/99 7 que regulamenta os medicamentos genéricos e que contempla a expansão do acesso por parte da população a medicamentos seguros, eficazes, com qualidade e a baixo custo, foi finalmente aprovada e a partir daí vem sendo implementada. A redução do preço final é possível, pois as indústrias produtoras de medicamentos genéricos não investem em propaganda em proporção similar àquela dos produtos de marca, além de não necessitarem realizar ensaios clínicos já feitos pelas indústrias detentoras das patentes ${ }^{8}$. Tal fato culmina com a diminuição do preço final nas farmácias da rede privada $\mathrm{e}$ dos gastos com medicamentos, por parte da rede pública de saúde, com a conseqüente racionalização de recursos dos programas de assistência farmacêutica oficiais 9 .

Por gerar polêmicas em torno da qualidade, segurança e eficácia dos medicamentos, a Lei $n^{\circ}$. 9.787/997, logo depois de iniciada sua implementação, passou a ser combatida pelas indústrias fabricantes de produtos de marca que objetivam manter o monopólio e a hegemonia das marcas comerciais. Os laboratórios farmacêuticos, na ocasião, manipularam a mídia com a finalidade de confundir a população e inviabilizar, impor obstáculos ou adiar a entrada em vigor das estratégias e diretrizes previstas na Lei ${ }^{9}$.

Para superar o monopólio dos titulares das patentes e estabelecer uma competição no mercado, via medicamentos genéricos, faz-se necessário o fortalecimento da política de "genéri- cos" a partir do compromisso dos atores sociais - profissionais de saúde, população e Estado - na divulgação das informações sobre as especialidades genéricas 10 .

O presente estudo tem como objetivo verificar o grau de conhecimento e informação sobre os medicamentos genéricos, em amostra representativa de usuários do serviço público de saúde ambulatorial do Recife, Pernambuco, Brasil e sua associação com o perfil sócio-econômico desses usuários.

\section{Métodos}

\section{Área estudada}

O estudo foi realizado na cidade do Recife, capital do Estado de Pernambuco. Possui 94 bairros distribuídos em seis distritos sanitários (DS) 11. Os serviços, em cada distrito de saúde, que apresentaram um grande número de pacientes atendidos no ambulatório, no ano de 2002, e que possuíam atendimento para clínica geral, foram os selecionados para a realização do estudo. Esses dados foram obtidos a partir do Sistema de Informação Ambulatorial do Sistema Único de Saúde (SIA/SUS). Estes serviços possuíam atendimento em clínicas especializadas (cardiologia, endocrinologia, ginecologia, psiquiatria).

\section{População estudada e coleta de dados}

Participaram da pesquisa os indivíduos maiores de 21 anos, atendidos pela clínica geral dos serviços selecionados para o estudo, residentes na cidade do Recife no ano de 2003 e que concordavam em assinar o Termo de Consentimento Livre e Esclarecido.

Foi realizado o estudo piloto $(n=177)$, no período de julho a agosto de 2003, com os pacientes dos setores de procedimentos ambulatoriais, ortopedia e clínica médica do Hospital das Clínicas da Universidade Federal de Pernambuco (HC-UFPE) e no setor de triagem clínica da Policlínica Amaury Coutinho que procuravam atendimento especializado. Este estudo objetivou identificar falhas no instrumento de coleta quanto à compreensão das perguntas pelos entrevistados, quanto às dificuldades e/ou facilidades do acesso às informações, quanto ao tempo necessário à coleta de dados na pesquisa definitiva $\mathrm{e}$ quanto à inclusão de perguntas no instrumento de coleta. Avaliado o instrumento de coleta ele foi considerado adequado à realização da pesquisa definitiva.

Tanto o projeto piloto como a pesquisa definitiva foram realizados pela pesquisadora em 
uma sala cedida pela diretoria do serviço de saúde. A investigação ocorreu no período de setembro a dezembro de 2003, no horário do atendimento com o clínico geral - turno da manhã - em todos os dias úteis da semana. Para evitar recusas quanto à participação no estudo, pois os pacientes eram chamados para o atendimento na clínica geral e não estando presentes eles perdiam o atendimento, a pesquisadora entregava fichas aos pacientes sendo a eles explicado que se tratava de uma entrevista sobre medicamentos genéricos. Aos entrevistados que compareciam à entrevista era explicado o objetivo da pesquisa. Ao concordarem em participar do estudo eles assinavam o Termo de Consentimento Livre e Esclarecido.

Uma amostra representativa da população usuária do serviço público ambulatorial de saúde dos DS I, II, III, IV, V e VI foi calculada segundo Moser \& Kalton 12 - intervalo de confiança de 95\% (IC95\%) e um poder de $80 \%$ - e se obteve um valor igual a 384 indivíduos. Este valor foi aproximado para 400 indivíduos.

Para determinar a distribuição da amostra entre os seis serviços de saúde selecionados o somatório do número de atendimentos ambulatoriais de cada serviço determinou um valor total (99.756 atendimentos) que foi utilizado na regra de três simples determinando um percentual referente ao número de indivíduos que deveriam ser entrevistados. Isto posto, para o serviço de saúde selecionado no DS I (16.270 atendimentos em 2002) o percentual foi igual a $16,3 \%$, para o DS II (33.697 atendimentos em 2002) foi de 33,7\%, para o DS III (12.291 atendimentos em 2002) foi de 12,3\%, para o DS IV (13.144 atendimentos em 2002) foi de $13,17 \%$, para o DS V (14.616 atendimentos em 2002) foi de $14,6 \%$ e para o DS VI (9.738 atendimentos em 2002) foi de 9,7\%. Foram abordadas 430 pessoas, destas 32 recusaram participar da investigação, representando um percentual de não respostas igual a $8 \%$. O estudo cumpre os princípios éticos contidos na Resolução no. 196/96 13 sobre pesquisa envolvendo seres humanos.

A presente investigação foi desenvolvida através de um estudo individualizado observacional transversal, também conhecido como estudo de prevalência 14 .

A entrevista foi realizada por meio de um questionário estruturado abordando os dados gerais do entrevistado e informações do entrevistado a respeito do objeto em estudo.

\section{Variáveis do estudo}

A escolha das variáveis para esclarecer o conhecimento e a informação da amostra sobre o medi- camento genérico foi aquela adotada na pesquisa nacional de opinião pública, com consumidores de medicamentos interceptados em drogarias no ano de 2001, realizada pela ANVISA que apreendeu o grau de conhecimento, informação e perfil de prescrição, dispensação e aquisição do medicamento genérico 6 .

No instrumento de coleta de dados foram elaboradas duas perguntas para mensurar a informação sobre a especialidade farmacêutica genérica: (1) "O Sr(a). já ouviu falar sobre o medicamento genérico?” (sim/não/não se aplica); (2) “Se conhece o medicamento 'genérico', quem informou?" (rádio, televisão, jornal/revista, médico, balconista, amigo/parente/vizinho). Para esta variável o entrevistado poderia responder a mais de uma alternativa que tinha resposta do tipo sim/não/não se aplica.

As perguntas que apontaram o conhecimento do entrevistado sobre o medicamento genérico foram: (1) "O Sr(a). conhece o medicamento genérico?” (sim/não/não se aplica); (2) "O Sr(a). sabe o que é um medicamento genérico?" (sim/ não/não se aplica); (3) "O que o Sr(a). entende por medicamento genérico?" (tem o mesmo efeito do medicamento de marca - sim/não/não se aplical não sabe; tem menor preço que o medicamento de marca - sim/não/não se aplica/não sabe; tem a mesma substância que o medicamento de marca - sim/não/não se aplica/não sabe); (4) "De que forma o Sr(a). identifica o medicamento genérico? (pelo 'G' impresso na caixa - sim/não/não se aplica/não sabe; pela tarja amarela na caixa com $o$ ' $G$ ' - sim/não/não se aplica/não sabe; pela indicação do número da Lei - sim/não/não se aplica/ não sabe; pelo preço - sim/não/não se aplica/não sabe; pelo nome da substância - sim/não/não se aplica/não sabe). Para quantificar a capacidade de identificação do medicamento genérico entre os entrevistados foi criada uma variável dicotômica do tipo "sim" e "não" - identificação do genérico.

As variáveis sócio-demográficas foram as seguintes: sexo, idade (anos completos), escolaridade, renda individual (em salários mínimos da época) e profissão.

Quanto às respostas "não sabe” foram assim consideradas quando os entrevistados não conseguiam opinar ou expressavam o seu desconhecimento. Em relação às respostas "não se aplica" foram tomadas aquelas em que o indivíduo não conseguia responder a uma pergunta anterior diretamente relacionada à posterior, por exemplo, "ter ouvido falar sobre o medicamento genérico" e "saber" e/ou "conhecer o medicamento genérico". 


\section{Análise dos dados}

Os dados foram computados no programa Epi Info versão 6.0 (Centers for Disease Control and Prevention, Atlanta, Estados Unidos). O teste do qui-quadrado de Mantel-Haenszel foi utilizado na análise bruta para comparação de proporções e, para ajustamento das razões, por análise estratificada das variáveis de potencial efeito modificador ou de confundimento, como o sexo, a idade e a renda. A regressão logística foi utilizada para obter estimativas de odds ratio e intervalos de confiança ajustados para as variáveis de confusão. Para testar o modelo de regressão logística foram incluídas as variáveis que apresentaram associação com "o conhecimento e a informação sobre o medicamento genérico" em nível de $\mathrm{p}<$ 0,20 na análise bivariada.

\section{Resultados}

A análise dos questionários evidenciou que $76,1 \%$ dos indivíduos eram do sexo feminino, $41,3 \%$ com idade superior a 50 anos (média = 47,5 anos), renda superior a um salário mínimo (51,4\%), porém, destes, $69,3 \%$ não trabalhavam. Entre os indivíduos que não se inseriam na população economicamente ativa, 39,8\% eram aposentados. Entre os 30,7\% que trabalhavam, $30,8 \%$ eram autônomos e $21,7 \%$ eram empregadas domésticas. Do total de entrevistados, $63 \%$ freqüentaram a escola por pelo menos nove anos (ensino fundamental completo ou incompleto), $10 \%$ eram analfabetos e destes $40 \%$ eram analfabetos funcionais (não freqüentaram a escola, mas sabiam ler ou escrever).

A análise evidenciou que $95,7 \%$ já ouviram falar a respeito do medicamento genérico, $68,1 \%$ sabiam identificá-lo e diferenciá-lo do medicamento de marca e $65,3 \%$ sabiam definir o medicamento genérico (Tabela 1).

Entre os 260 indivíduos que sabiam definir o medicamento genérico, $40 \%$ o definiram como aquele que possui menor preço que a marca de referência. Quanto à forma de identificação do medicamento genérico, $47 \%$ apontaram a presença da letra $\mathrm{G}$ na embalagem do produto, enquanto que $25 \%$ afirmaram identificá-lo pela letra $\mathrm{G}$ contida na tarja amarela. A partir da criação da variável identificação do medicamento genérico foi evidenciado que $91,5 \%$ dos entrevistados eram capazes de reconhecer o medicamento genérico.

Na Figura 1 está apresentado o percentual de indivíduos que identificaram o produto genérico pelo preço e pela substância com o percentual de indivíduos que o definiram pelo menor preço que a marca e por possuir a mesma substância que ela. Foi verificado que dos 186 (46,7\%) que definiram o produto genérico como tendo a mesma substância que o de marca comercial, somente $19,4 \%$ reconheciam-no pelo nome do princípio ativo e dos 273 (68,9\%) entrevistados que afirmaram ter o medicamento genérico menor preço que o de marca comercial, apenas

Tabela 1

Freqüência da informação (ouviu falar sobre o medicamento genérico) e do conhecimento

(conhecer o medicamento genérico, saber o que é um medicamento genérico e identificar o medicamento genérico)

na amostra estudada. Recife, Pernambuco, Brasil, setembro a dezembro de 2003.

\begin{tabular}{lcc}
\hline Variável & Frequência & $\%$ \\
\hline Ouviu falar sobre o medicamento genérico & & \\
Sim & 381 & 95,7 \\
Não & 17 & 4,3 \\
Conhece o medicamento genérico & 271 & 68,1 \\
Sim & 127 & 31,9 \\
Não & & 65,3 \\
Sabe definir o medicamento genérico & 260 & 34,7 \\
Sim & 138 & 91,5 \\
Não & & 8,5 \\
Identificação do medicamento genérico & 364 & 100,0 \\
Sim & 34 & 398 \\
$\quad$ Não & & \\
Total & & \\
\hline
\end{tabular}


Figura 1

Comparação entre identificar o medicamento genérico como possuindo a mesma substância e menor preço que o medicamento de marca comercial e defini-lo por estas mesmas características. Recife, Pernambuco, Brasil, outubro a dezembro de 2003.

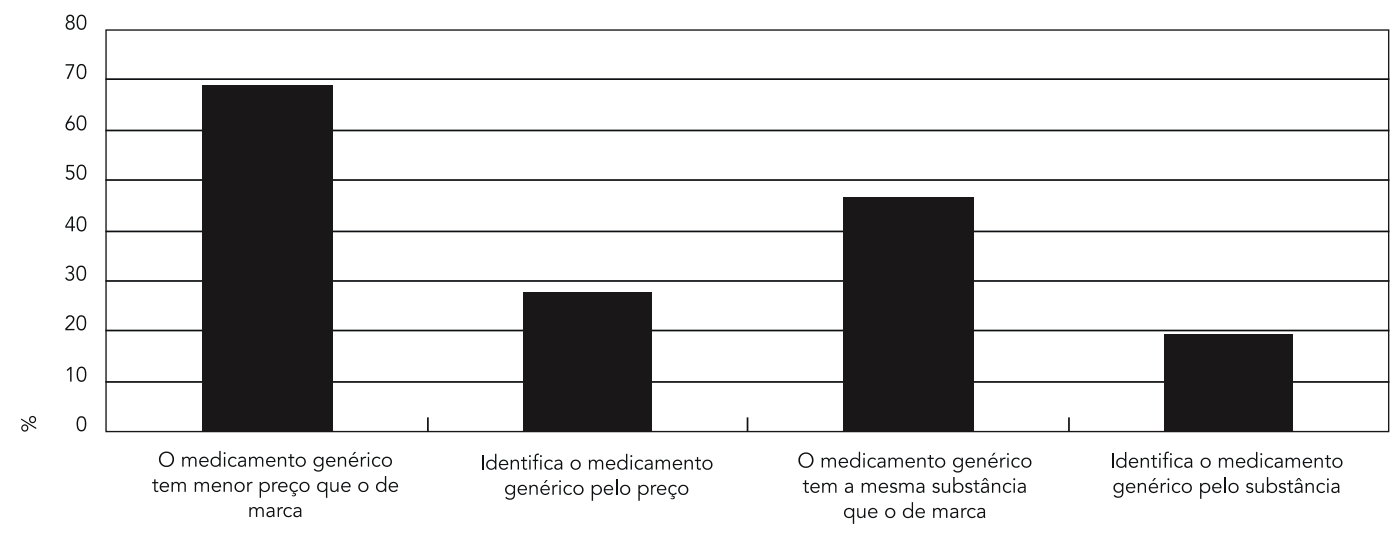

27,8\% mencionaram reconhecê-lo pelo preço (Figura 1).

No que tange ao meio de comunicação pelo qual obtiveram informação sobre o "genérico", $32 \%$ citaram a televisão, seguida pelo rádio, $17 \%$ (Figura 2).

Para demonstrar a influência de algumas variáveis sócio-demográficas quanto à informação e ao conhecimento do medicamento genérico foi realizada análise bruta. Na Tabela 2 está evidenciado que o sexo, a idade e a renda, na análise bruta e ajustada, não apresentaram associações significantes $(p<0,05)$ com a variável de informação. Entretanto, a escolaridade, na análise bruta e ajustada, apresentou um nível de significância estatística $-\mathrm{p}=0,048$ - apesar da diferença entre os grupos ser aparentemente igual. $\mathrm{O}$ conhecimento do medicamento genérico está estatisticamente associado à escolaridade como também à idade, tanto na análise bruta quanto na ajustada.

Quanto a saber o que é o medicamento genérico, analisada como uma variável que mensurou o conhecimento, foi verificada associação estatisticamente significante com a escolaridade e com a idade $\left(\chi^{2}=19,86 ; p<0,05\right)$ tanto na análise bruta quanto na ajustada. Esta relação é mais visível nos indivíduos de faixa etária menor que cinqüenta anos cuja probabilidade de saberem o que é um medicamento genérico foi 1,28 vezes maior do que a faixa etária igual ou maior que cinqüenta anos. Ao compararmos os indivíduos que possuíam 12 anos de estudo ou mais com os que cursaram menos de 12 anos, foi verificado
Figura 2

Distribuição dos meios que informaram sobre a existência do medicamento genérico Recife, Pernambuco, Brasil, setembro a dezembro de 2003

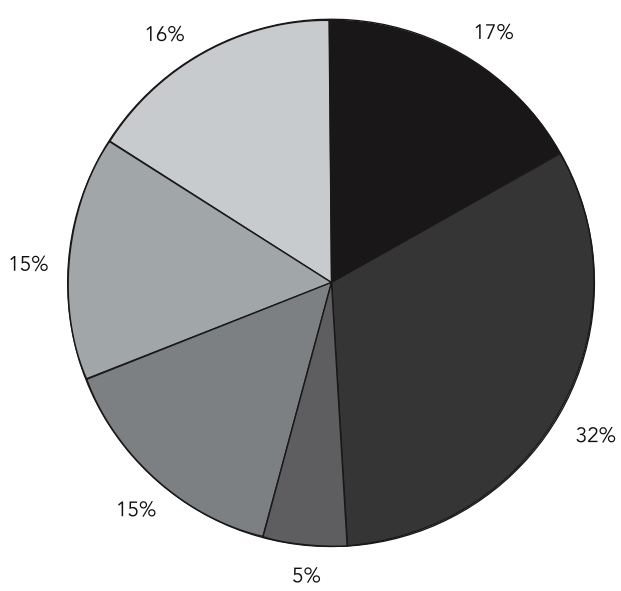

Rádio Televisão Jornal/Revista Médico Balconista Conhecido

que a probabilidade dos primeiros saberem o que é um medicamento genérico foi 6,15 vezes maior do que os segundos. Aqueles, quando comparados aos analfabetos, tinham uma probabilidade 1,53 vezes maior. De acordo com o p valor, existe uma associação estatisticamente significante $\left(\chi^{2}=94,31 ; p<0,05\right)$ (Tabela 3$)$. 
Quanto ao meio de obtenção de informação sobre o genérico, a análise estratificada por sexo, idade, renda e escolaridade demonstrou que ambos os sexos apontaram a televisão (82,1\% dos homens e $85,8 \%$ das mulheres) como o principal meio de informação, como também ambas as faixas etárias, tanto os indivíduos com renda inferior quanto os com renda superior a um salá- rio mínimo e entre os analfabetos e aqueles que freqüentaram a escola por períodos inferiores ou superiores a 12 anos.

Foi observada, também, uma relação estatisticamente significante $\left(\chi^{2}=5,24 ; p<0,05\right)$ entre possuir renda menor que um salário mínimo e ser a televisão o meio que informa sobre o medicamento genérico. Evidenciou-se uma asso-

Tabela 2

Freqüência da informação (ter ouvido falar sobre o medicamento genérico) e do conhecimento (conhecer o medicamento genérico)

segundo sexo, idade, renda e escolaridade. Recife, Pernambuco, Brasil, setembro a dezembro de 2003.

\begin{tabular}{|c|c|c|c|c|c|c|c|c|}
\hline \multirow[t]{2}{*}{ Variável } & \multicolumn{4}{|c|}{ Informação } & \multicolumn{4}{|c|}{ Conhecimento } \\
\hline & $\mathrm{P}(\%)$ & RP & IC95\% & $p$ valor & $\mathrm{P}(\%)$ & RP & IC95\% & $p$ valor \\
\hline \multicolumn{9}{|l|}{ Sexo } \\
\hline Masculino & 96 & 1,00 & & 0,58 & 68 & 1,00 & & 0,67 \\
\hline Feminino & 95 & 0,99 & $0,94-1,04$ & & 66 & 0,97 & $0,82-1,14$ & \\
\hline \multicolumn{9}{|c|}{ Idade (anos completos) } \\
\hline$\geq 50$ & 94 & 1,00 & & 0,13 & 60 & 1,00 & & 0,003 \\
\hline$<50$ & 97 & 1,03 & $0,99-1,08$ & & 74 & 1,23 & $1,07-1,43$ & \\
\hline \multicolumn{9}{|c|}{ Renda (salário mínimo) } \\
\hline$<1$ & 97 & 1,00 & & 0,10 & 70 & 1,00 & & 0,70 \\
\hline$\geq 1$ & 94 & 0,96 & 0,99-1,08 & & 67 & 0,95 & $0,90-1,17$ & \\
\hline \multicolumn{9}{|c|}{ Escolaridade (anos de estudo) } \\
\hline Analfabeto & 90 & 1,00 & & 0,048 & 42 & 1,00 & & 0,0005 \\
\hline$<12$ & 96 & 1,06 & $0,95-1,18$ & & 67 & 1,59 & $1,10-2,31$ & \\
\hline$\geq 12$ & 99 & 1,10 & $0,99-1,22$ & & 81 & 1,92 & $1,31-2,77$ & \\
\hline
\end{tabular}

P: prevalência; RP: razão de prevalência.

\begin{tabular}{|c|c|c|c|c|}
\hline Variável & $\mathbf{P}(\%)$ & IC95\% & RP & $p$ valor \\
\hline \multicolumn{5}{|l|}{ Sexo } \\
\hline Masculino & 66 & & 1,00 & 0,62 \\
\hline Feminino & 63 & $0,80-1,14$ & 0,95 & \\
\hline \multicolumn{5}{|c|}{ Idade (anos completos) } \\
\hline$\geq 50$ & 56 & & 1,00 & 0,0009 \\
\hline$<50$ & 72 & $1,10-1,50$ & 1,28 & \\
\hline \multicolumn{5}{|c|}{ Renda (salário mínimo) } \\
\hline$<1$ & 70 & & 1,00 & 0,13 \\
\hline$\geq 1$ & 62 & $0,97-1,29$ & 1,12 & \\
\hline \multicolumn{5}{|c|}{ Escolaridade (anos de estudo) } \\
\hline Analfabeto & 52 & & 1,00 & 0,000 \\
\hline$<12$ & 13 & $0,15-0,45$ & 0,25 & \\
\hline$\geq 12$ & 80 & $1,12-2,08$ & 1,53 & \\
\hline
\end{tabular}

P: prevalência; RP: razão de prevalência. 
ciação estatisticamente significante $\left(\chi^{2}=32,53\right.$; $\mathrm{p}<0,05$ ) entre ter freqüentado a escola por $12 \mathrm{ou}$ mais anos e obter informações no jornal ou em revistas (Tabela 4 ).

Ao final da regressão múltipla, ficou demonstrado que no caso do conhecimento do genérico $(\mathrm{n}=390)$ a escolaridade e a faixa etária foram utilizadas na análise de regressão logística que evidenciou somente a influência da escolaridade como determinante do conhecimento (Tabela 5).

\section{Discussão}

Segundo Pinheiro et al. 15 que efetuou uma análise sobre o acesso e uso dos serviços de saúde entre homens e mulheres no Brasil, foi verificado que $75 \%$ das mulheres que residem na zona urbana utilizam com maior freqüência os serviços de saúde por possuírem maior preocupação com a saúde, como também, a existência de diversos programas de saúde (pré-natal, prevenção de câncer de colo uterino e da mama) voltados para este grupo, possibilitando a sua maior medicalização, além de justificar o alto percentual de mulheres no presente estudo $(76,1 \%)$.

Pinheiro et al. 15 também citam a faixa etária entre 25 a 65 anos ou mais - acima de $65 \%$ - como aquela que regularmente utiliza o serviço de saúde. Este dado também se coaduna com os resultados observados no presente estudo, o qual demonstrou que 41,3\% dos usuários do serviço

Tabela 4

Freqüência dos meios de obtenção de informação sobre o medicamento genérico segundo as variáveis sexo,

idade, renda e escolaridade. Recife, Pernambuco, Brasil, setembro a dezembro de 2003.

\begin{tabular}{|c|c|c|c|c|c|c|c|c|c|c|c|c|}
\hline \multirow[t]{2}{*}{ Variável } & \multicolumn{4}{|c|}{ Televisão } & \multicolumn{4}{|c|}{ Rádio } & \multicolumn{4}{|c|}{ Jornal/Revista } \\
\hline & $\mathbf{P}(\%)$ & RP & IC95\% & $p$ valor & $\mathrm{P}(\%)$ & RP & IC95\% & $p$ valor & $\mathrm{P}(\%)$ & RP & IC95\% & p valor \\
\hline \multicolumn{13}{|l|}{ Sexo } \\
\hline Masculino & 82,1 & 1,00 & $0,86-1,06$ & 0,37 & 54,7 & 1,00 & $1,05-1,65$ & 0,02 & 22,1 & 1,00 & $1,52-4,36$ & 0,00 \\
\hline Feminino & 85,8 & 1,04 & & & 41,6 & 0,76 & & & 8,60 & 0,38 & & \\
\hline \multicolumn{13}{|c|}{ Idade (anos completos) } \\
\hline$\geq 50$ & 79,3 & 1,00 & $1,02-1,23$ & 0,008 & 48,8 & 1,00 & $0,69-1,06$ & 0,15 & 8,50 & 1,00 & $0,92-3,00$ & 0,08 \\
\hline$<50$ & 88,8 & 1,12 & & & 41,6 & 0,85 & & & 14,2 & 1,67 & & \\
\hline \multicolumn{13}{|c|}{ Renda (salário mínimo) } \\
\hline$<1$ & 89,1 & 1,00 & $1,01-1,20$ & 0,02 & 42,2 & 1,00 & $0,72-1,12$ & 0,35 & 10,9 & 1,00 & $0,51-1,53$ & 0,67 \\
\hline$\geq 1$ & 80,8 & 0,91 & & & 46,8 & 1,11 & & & 12,3 & 1,13 & & \\
\hline \multicolumn{13}{|c|}{ Escolaridade (anos de estudo) } \\
\hline Analfabeto & 82,5 & 1,00 & & 0,07 & 42,5 & 1,00 & & 0,54 & 5,0 & 1,00 & & 0,00 \\
\hline$<12$ & 82,5 & 1,00 & $0,86-1,17$ & & 42,6 & 1,00 & $0,68-1,48$ & & 6,77 & 1,35 & $0,33-5,64$ & \\
\hline$\geq 12$ & 92,0 & 1,11 & $0,96-1,30$ & & 49,0 & 1,15 & $0,76-1,74$ & & 28,0 & 5,60 & $1,40-22,4$ & \\
\hline \multirow[t]{2}{*}{ Variável } & \multicolumn{4}{|c|}{ Médico } & \multicolumn{4}{|c|}{ Balconista } & \multicolumn{4}{|c|}{ Conhecidos } \\
\hline & $\mathrm{P}(\%)$ & RP & IC95\% & $p$ valor & $\mathrm{P}(\%)$ & RP & IC95\% & $\mathrm{p}$ valor & $\mathrm{P}(\%)$ & RP & IC95\% & $p$ valor \\
\hline \multicolumn{13}{|l|}{ Sexo } \\
\hline Masculino & 35,8 & 1,00 & $0,69-1,27$ & 0,66 & 36,8 & 1,00 & $0,70-1,26$ & 0,67 & 34,7 & 1,00 & $0,58-1,06$ & 0,10 \\
\hline Feminino & & 38,3 & 1,06 & & & 39,3 & 1,06 & & & 44,2 & 1,27 & \\
\hline \multicolumn{13}{|c|}{ Idade (anos completos) } \\
\hline$\geq 50$ & 36,0 & 1,00 & $0,83-1,39$ & 0,59 & 34,1 & 1,00 & $0,95-1,60$ & 0,11 & 40,2 & 1,00 & $0,85-1,37$ & 0,53 \\
\hline$<50$ & & & 38,6 & 1,07 & & & 42,1 & 1,23 & & & 43,3 & 1,07 \\
\hline \multicolumn{13}{|c|}{ Renda (salário mínimo) } \\
\hline$<1$ & 37,0 & 1,00 & $0,75-1,24$ & 0,77 & 40,1 & 1,00 & $0,85-1,39$ & 0,52 & 44,8 & 1,00 & $0,92-1,47$ & 0,19 \\
\hline$\geq 1$ & & 38,4 & 1,04 & & & 36,9 & 0,92 & & & 38,4 & 0,85 & \\
\hline \multicolumn{13}{|c|}{ Escolaridade (anos de estudo) } \\
\hline Analfabeto & 25,0 & 1,00 & & 0,19 & 30,0 & 1,00 & & 0,35 & 35,0 & 1,00 & & 0,59 \\
\hline$<12$ & 38,2 & 1,53 & $0,87-2,68$ & & 38,2 & 1,27 & $0,77-2,10$ & & 43,4 & 1,24 & $0,79-1,94$ & \\
\hline$\geq 12$ & 41,0 & 1,64 & $0,91-2,95$ & & 43,0 & 1,43 & $0,85-2,42$ & & 41,0 & 1,17 & $0,72-1,90$ & \\
\hline
\end{tabular}

P: prevalência; RP: razão de prevalência. 
Tabela 5

Análise multivariada da escolaridade associada ao conhecimento do medicamento genérico ( $n=390)$. Recife, Pernambuco, Brasil, setembro a dezembro de 2003.

\begin{tabular}{lccc}
\hline Variável & OR & IC95\% & p valor \\
\hline $\begin{array}{l}\text { Escolaridade (anos de estudo) } \\
\quad<12\end{array}$ & 0,35 & & \\
$\geq 12$ & 0,17 & $0,18-0,70$ & 0,0028 \\
& $0,07-0,38$ & 0,000 \\
\hline
\end{tabular}

público de saúde com idade superior a cinqüenta anos de idade são usuários do serviço de saúde.

Os resultados da renda individual vislumbrados $-51,4 \%$ com renda superior a um salário mínimo - aproximam-se da média da Região Nordeste para o ano de 1999 (52,4\% com renda superior a um salário mínimo - igual a 151,00 reais) 16. A renda referida por $44,2 \%$ dos entrevistados que recebiam até 0,5 salário mínimo em 2003 é inferior ao salário mínimo no ano de 1999 - devido ao grande percentual (69,3\%) de indivíduos que não trabalhavam e, conseqüentemente, sem uma renda fixa mensal. O percentual de autônomos $(30,8 \%)$ reflete o tipo de vínculo empregatício frágil para estes cujos direitos trabalhistas e sociais requerem revisão urgente e para aqueles que não gozam de nenhum tipo de benefício previsto em nossa lei trabalhista.

O baixo grau de instrução (63\% tinham até o ensino fundamental) ainda é marcante entre os usuários do serviço público de saúde, o que impede que esses indivíduos, sendo desconhecedores de seus direitos constitucionais em relação à saúde, deixem de reivindicar por melhores condições de assistência à saúde. Vale salientar que os indivíduos desprovidos de conhecimento apresentam dificuldades de entendimento quanto à correta e racional utilização dos medicamentos, da orientação prestada pelo profissional de saúde e da compreensão sobre o seu estado de saúde.

A ANVISA, no ano de 2001, entrevistou, em drogarias, 2.200 consumidores de medicamentos e verificou que $95 \%$ dos entrevistados conheciam os medicamentos genéricos, $91 \%$ o definiram corretamente e que $71 \%$ reconheciam-no pela letra $\mathrm{G}$ da embalagem ${ }^{6}$. Tais achados não demonstram os mesmos resultados, apesar do público ter sido o mesmo - consumidores de medicamentos - porém, sendo a pesquisa realizada em ambientes diferentes, pois na drogaria, provavelmente, $o$ consumidor, no momento do contato com o balconista da farmácia, fora apresentado ao produto genérico, fato que não ocorreu com os usuários do serviço de saúde cujos comentários a respeito do genérico não foram realizados pela pesquisadora. Daí as diferenças quanto aos percentuais das variáveis conhecer e saber definir. O mesmo não se verifica quanto à identificação do genérico cujo percentual foi idêntico em ambos os estudos. $\mathrm{O}$ fato de saber definir o medicamento genérico confere ao indivíduo segurança quanto ao uso de um produto de mesma substância e efeito terapêutico do medicamento de marca comercial.

Ao confrontar o percentual de indivíduos que identificaram o produto genérico pelo preço e pela substância com o percentual de indivíduos que o definiram pelo menor preço que a marca e por possuir a mesma substância que ela, foi verificada a existência de inconsistências, pois dos $186(46,7 \%)$ que definiram o produto genérico como tendo a mesma substância que o de marca comercial, somente $19,4 \%$ reconheciam-no pelo nome do princípio ativo e dos 273 (68,9\%) entrevistados que afirmaram ter o medicamento genérico menor preço que o de marca comercial, apenas $27,8 \%$ mencionaram reconhecê-lo pelo preço.

Um estudo realizado em Porto Alegre, Rio Grande do Sul, Brasil, com 124 pessoas objetivou avaliar o conhecimento sobre a composição de medicamentos e constatou que $40 \%$ não sabiam nada sobre o assunto, demonstrando total desconhecimento sobre que tipo de substância ativa estava presente no medicamento e qual o seu efeito terapêutico ${ }^{17}$. Sabe-se que a população faz uso de vários medicamentos com diferentes marcas comerciais, mas possuidores do mesmo princípio ativo, o que expõe ao risco de superdosagem, efeitos colaterais e interações medicamentosas 17 .

Os resultados confirmaram que os meios de comunicação de massa constituem o veículo adequado para informar a população sobre os medicamentos genéricos. A televisão foi o principal meio de informação entre os indivíduos com idade inferior a cinqüenta anos e com renda abaixo de um salário mínimo. Apesar de a televisão ter sido apontada como a que informa sobre o "genérico", dado interessante foi o que apontou a informação em jornal e/ou revista entre aqueles com maior escolaridade. Quanto mais anos de estudo, maior o conhecimento a respeito do produto genérico $\left(\chi^{2}=19,86 ; \mathrm{p}<0,05\right)$. A faixa etária de menores de cinqüenta anos apresentou maior grau de conhecimento - probabilidade 1,23 vez maior - do que aqueles com idade igual ou superior a cinqüenta anos $\left(\chi^{2}=8,75 ; \mathrm{p}<0,05\right)$. Como confirmado em vários estudos, a escolaridade é uma variável que influencia o entendimento e a compreensão do que vem a ser a especialidade farmacêutica genérica 18 . 
Ressaltemos, ademais, que a troca de informações entre o prescritor e o paciente determinará a indicação do medicamento a ser prescrito. Neste ciclo não se pode descartar a contribuição do farmacêutico como ator que provê informações sobre o uso do medicamento - possíveis interações, efeitos adversos, efeito terapêutico, dose do medicamento, posologia, cuidados especiais no armazenamento. $\mathrm{O}$ acúmulo destes conhecimentos pelo paciente, favorece a assimilação de informações a respeito do produto - efeito terapêutico, contra-indicações, efeitos colaterais. Adicionalmente, entre outros aspectos, o grau de conhecimento melhora a interação médico-paciente-farmacêutico 19.

Entretanto, segundo Silva et al. 20 que determinaram o grau de conhecimento sobre itens como dose e posologia para o uso seguro de medicamentos em pacientes ambulatoriais, apesar de o conhecimento ser suficiente ainda é necessária a implementação das informações dadas ao paciente a respeito do medicamento.

\section{Conclusão}

Foi constatado que a maioria dos usuários do serviço público de saúde ambulatorial do Recife são do sexo feminino com idade média de 47,5 anos e analfabetos.

Depois de transcorrido um quadriênio da introdução dos medicamentos genéricos no mercado brasileiro, no presente estudo se constatou que a grande maioria dos usuários tinha um bom grau de informação sobre o genérico, no que diz respeito a "ter ouvido falar", "conhecer esta especialidade farmacêutica" e "saber o que é o produto genérico".

A escolaridade demonstrou está associada positivamente às variáveis conhecer e entender o que é o medicamento genérico estando esta última também associada à faixa etária de menores de cinqüenta anos. Daí o destaque para o desenvolvimento de ações educativas indepen- dentes que informem à população da existência de produtos idênticos em qualidade, segurança e eficácia ao de marca comercial, no entanto, com menor custo para sua aquisição, o que contribui à realização ou manutenção de uma farmacoterapia integral.

Ao identificar o preço do medicamento genérico como um importante item para a sua identificação, como também o foi a "tarja amarela” com a letra $\mathrm{G}$, é preciso dar um maior destaque ao reconhecimento do produto genérico pelo nome da substância ativa presente na embalagem do produto. A publicidade maciça desenvolvida pelo Ministério da Saúde sobre a embalagem das especialidades farmacêuticas genéricas foi amplamente apreendida pela televisão e pelo rádio.

No que se refere aos aspectos metodológicos, a etapa de planejamento do estudo buscou evitar vieses: identificação dos serviços de saúde de maior número de atendimentos ambulatoriais, seleção aleatória de participantes, testagem do instrumento de coleta de dados (estudo piloto), padronização dos procedimentos de coleta de dados e estímulo dos participantes a colaborar no estudo para garantir a validade interna. Apesar da garantia de que nenhum participante perderia a sua consulta médica, foi possível observar que este foi o principal motivo da recusa ( $8 \%$ de 430 indivíduos abordados). As respostas fornecidas pelos entrevistados foram consideradas verdadeiras tendo em vista a impossibilidade de validar estas informações devido ao período de tempo disponível.

Ainda que o uso adequado dos medicamentos e a disponibilidade dos mesmos, quando necessário, para todos, requeiram a implementação de um amplo conjunto de medidas, certamente, o programa de genéricos tem uma contribuição importante a dar, nesse contexto, e, apesar do relativamente pouco tempo de sua existência no país, já é possível asseverar terem sido dados passos significativos para a consolidação do mencionado programa. 


\section{Resumo}

A Lei no. 9.787/99, que regulamenta os medicamentos genéricos, contempla normas para o registro e prevê a expansão do acesso da população a medicamentos seguros, eficazes, com qualidade e baixo custo. Após um quadriênio da introdução dos genéricos no Brasil, propõe-se verificar o conhecimento e a informação sobre genéricos entre os usuários dos serviços públicos de saúde ambulatorial do Recife, Pernambuco. No ano de 2002, foi aplicado um questionário pré-elaborado. Dos entrevistados, $76,1 \%$ eram do sexo feminino. Ouviram falar sobre o "medicamento genérico" 95,7\% e 68,1\% conheciam o medicamento genérico. As ações adotadas pelo governo, quanto à divulgação das propriedades desta especialidade farmacêutica e às vantagens oferecidas ao consumidor, atingiram sobremaneira esta população.

Medicamentos Genéricos; Conhecimento; Informação; Serviços Ambulatoriais de Saúde

\section{Referências}

1. Dighe SV. A review of the safety of generic drugs. Transplant Proc 1999; 31:23S-4S

2. Silveira FG, Osório RG, Piola SF. Os gastos familiares com saúde. Ciênc Saúde Coletiva 2002; 7:71931.

3. Barros JAC. Diálogo de surdos. Jornal do Commércio 2000; 28 mar.

4. King DR, Kanavos P. Encouraging the use of generic medicines: implications for transition economies. Croat Med J 2002; 43:462-9.

5. Brasil. Portaria no. 3.916. Política Nacional de Medicamentos. Diário Oficial da União 1998; 30 out.

6. Agência Nacional de Vigilância Sanitária. Medicamentos genéricos no Brasil: pesquisa com consumidores. http://www.anvisa.org.br (acessado em 12/Ago/2002).

7. Brasil. Lei no. 9.787. Política de Medicamentos Genéricos. Diário Oficial da União 1999; 10 fev.

8. Meadows M. Greater access to generic drugs. New FDA initiatives to improve drug reviews and reduce legal loopholes. FDA Consum 2003; 37:12-7.

9. Barros JAC. Similares $x$ genéricos. Jornal do Commércio 1999; $14 \mathrm{dez}$

10. Garattini L, Tediosi F. A comparative analysis of generics markets in five European countries. Health Policy 2000; 51:149-62.

11. Instituto Brasileiro de Geografia e Estatística. Cidades. http://www.ibge.gov.br/cidadesat/default. php (acessado em 10/Jun/2003).

12. Moser A, Kalton G. Survey methods in social investigation. 2nd Ed. London: Heinemann Press; 1985.

13. Conselho Nacional de Saúde. Resolução no. 196/96. Diretrizes e normas regulamentadoras de pesquisa envolvendo seres humanos. Diário Oficial da União 1996; 16 out.

\section{Colaboradores}

C. E. Rocha colaborou na revisão de literatura, elaboração da metodologia, análise dos resultados e redação do artigo final. J. A. C. Barros contribuiu na revisão de literatura, análise dos resultados e revisão do artigo final. M. D. P. Silva participou da elaboração da metodologia, análise dos resultados e revisão do artigo final.

\section{Agradecimentos}

Ao apoio financeiro da Coordenação de Aperfeiçoamento de Pessoal de Nível Superior e Fundação de Amparo à Ciência e Tecnologia do Estado de Pernambuco.

14. Hennekens CH, Burning JE. Epidemiology in medicine. Boston: Little, Brown and Company; 1987.

15. Pinheiro RS, Viacava F, Travassos C, Brito AS. Gênero, morbidade, acesso e utilização de serviços de saúde no Brasil. Ciênc Saúde Coletiva 2002; 7:6870707.

16. Departamento Intersindical de Estatística e Estudos Sócio-Econômicos. 1 de maio - dia do trabalho. A questão do salário mínimo. Boletim DIEESE. http://www.dieese.org.br/esp/bolsalmin.pdf (acessado em 15/Mai/2004).

17. Tierling VL, Paulino MA, Fernandes LC, Schenkel EP, Mengue SS. Nível de conhecimento sobre a composição de analgésicos com ácido acetilsalicílico. Rev Saúde Pública 2004; 38:223-7.

18. Gaither CA, Kirking DM, Ascione FJ, Welage LS. Consumer's views on generic medications. J Am Pharm Assoc 2001; 41:729-36.

19. Pepe VLE, Castro CGSO. A interação entre prescritores, dispensadores e paciente: informação compartilhada como possível benefício terapêutico. Cad Saúde Pública 2000; 16:815-22.

20. Silva T, Schenkel EP, Mengue SS. Nível de informação a respeito de medicamentos prescritos a pacientes ambulatoriais de hospital universitário. Cad Saúde Pública 2000; 16:449-55.

Recebido em 28/Abr/2005

Versão final reapresentada em 23/Fev/2006

Aprovado em 26/Mai/2006 